\title{
Microprocessing of Arched Bridge Structures with Epoxy Resin by Proton Beam Writing
}

\author{
Katsuyoshi Takano ${ }^{\mathrm{a}}$, Atsushi Asano ${ }^{\mathrm{a}}$, Yuta Maeyoshi ${ }^{\mathrm{a}}$, Hiromi Marui ${ }^{\mathrm{a}}$, Masaaki Omichi ${ }^{\mathrm{a}}$, \\ Akinori Saeki $^{a}$, Shu Seki ${ }^{a}$, Takahiro Satoh ${ }^{\text {b }}$, Yasuyuki Ishii ${ }^{b}$, Tomihiro Kamiya ${ }^{b}$, Masashi Koka ${ }^{b}$, \\ Takeru Ohkubo ${ }^{b}$, Masaki Sugimoto ${ }^{c}$ and Hiroyuki Nishikawa ${ }^{d}$ \\ ${ }^{a}$ Division of Applied Chemistry, Graduate School of Engineering, Osaka University, \\ 2-1 Yamadaoka, Suita, Osaka 565-0871, Japan \\ ${ }^{b}$ Takasaki Advanced Radiation Research Institute, Japan Atomic Energy Agency, \\ 1233 Watanuki-machi, Takasaki, Gunma 370-1292, Japan \\ ${ }^{c}$ Quantum beam directorate, Japan Atomic Energy Agency, \\ 1233 Watanuki-machi, Takasaki, Gunma 370-1292, Japan \\ ${ }^{d}$ Department of Electrical Engineering, Shibaura Institute of Technology, \\ 3-7-5 Toyosu, Koto-ku, Tokyo 135-8548, Japan \\ takano@chem.eng.osaka-u.ac.jp
}

Keywords: proton beam writing, photolithography, three dimensional structure, supercritical drying, $\mathrm{SU}-8$ photoresist

\section{Introduction}

The proton beam writing (PBW) with the energy with several $\mathrm{MeV}$ ranges has been expected to be a unique technique for three dimensional microprocessing with high aspect ratio for polymer materials in the scale from nano to micro meters [1-5]. With only one development on photolithographic technique, overhang or bridge like structures can be fabricated by multiple exposures with changing the beam energy, which corresponds to adjust the processing depth.

On the other hand, the supercritical drying using supercritical carbon dioxide $\left(\mathrm{CO}_{2}\right)$ under high pressure has been proposed as new drying method for a fabrication of the nano- and microstructures with high aspect ratio to avoid the pattern collapse of the structures made up of silicon or photoresist [6-8]. However, the supercritical drying for the resist patterns has not been widely applied. Because swelling effects for the resist patterns have not been clearly understood.

A swelling of polymer matrix with the free volume or uncrosslinking induced by the high pressure $\mathrm{CO}_{2}$ has been known $[9,10]$. The swelling is a problem for the resist patterning at the supercritical drying. But, utilizing a difference of the swelling ratio in the polymer matrix at the supercritical drying, a fabrication of curved structures is expected. In this study, fabrication of arched bridges of epoxy resin was tried by a combination of double exposure PBW with two kinds of energies and the supercritical drying after the development. A naturally drying was also performed to compare with the case of the supercritical drying. The swelling effect at the drying was investigated from a surface observation by Scanning Electron Microscopy (SEM), and discussed about the forming of arched shape.

\section{Method}

The epoxy films which consist of epoxy gel layer with $20 \mu \mathrm{m}$ thickness on an epoxy sheet were fabricated by spin-coat method without the pre-baking, using SU-8 2015 photoresist produced by MicroChem Co. Ltd. The epoxy sheets were fabricated by curing the SU-8 photoresist from irradiation of electron beam with the incident energy of $0.5 \mathrm{MeV}$ or ${ }^{60} \mathrm{Co}$ gamma ray at JAEA/Takasaki.

The double exposure PBW was performed with a light ion microbeam system connected to the 3 MV single-ended electrostatic accelerator at Takasaki Ion Accelerators for Advanced Radiation Application facility (TIARA), of JAEA/Takasaki [11]. On this PBW system, the overwriting to the replaced samples after the changing of the incident energy can be executed is the accuracy of the less than $5 \mu \mathrm{m}$ [5]. The proton beam was focused to $1 \mathrm{X}$ 
(a)

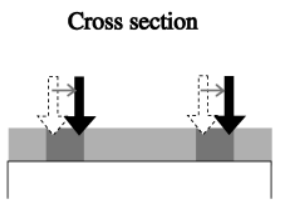

(b)

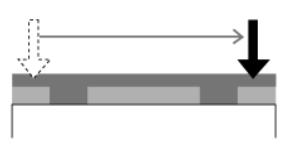

Top view
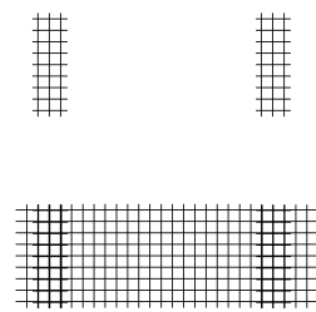

Fig. 1. Scheme of the double exposure PBW. The left is cross section of the sample. Pale gray rectangle is processing layer of SU-8 photoresist gel, and under white rectangle is the epoxy substrate which consists of cured SU-8 photoresist. Dark and dashed-outline arrows denote the injected ion beam and past one. Pale arrows indicate the track of beam sweeping. The dark gray tones denote the induced cross-linking along the ion trajectories. The right is trace of written patterns at the each procedure to see in the top view of the sample. (a) shows the pier patterns writing with $3 \mathrm{MeV}$ proton beam. (b) shows the girder pattern writing with $0.5 \mathrm{MeV}$ proton beam aiming at the pier pattern.

$1 \mu \mathrm{m}^{2}$ size in horizontal and vertical directions. The size of proton beam was estimated in the Full Width at Half Maximum (FWHM) from fitting Gaussian at the peak of the secondary electron yield, which was traced by line scanning of the focused beam across sharp edges on the cupper mesh. A current of proton beam was adjusted to be $10 \mathrm{pA}$, measuring with a Faraday cup at the sample position. A fluence of focused proton beam was adjusted by controlling an exposure time and a writing repetition.

The procedure scheme is shown in Fig. 1. At the first, pier patterns with a lattice were written at the beam energy of $3 \mathrm{MeV}$ at the fluence of $4.5 \mathrm{x}$ $10^{3}$ ions $/ \mu \mathrm{m}^{2}$. The penetration range of $3 \mathrm{MeV}$ proton for SU-8 photoresist with the density of 1.2 X $10^{3} \mathrm{~kg} / \mathrm{m}^{3}$ was estimated to be $125 \mu \mathrm{m}$ by a calculation using TRIM code [12]. Therefore, the protons penetrate the coated SU-8 photoresist layer.

The next, a girder pattern with a lattice were overwritten with $0.5 \mathrm{MeV}$ proton beam on the pier patterns at the fluence of $4.5 \times 10^{2}$ ions $/ \mu \mathrm{m}^{2}$. At the $0.5 \mathrm{MeV}$, the penetration range was estimated to be $7 \mu \mathrm{m}$. Therefore, the injected $0.5 \mathrm{MeV}$ protons

stopped at the depth of $7 \mu \mathrm{m}$ from the film surface.

To confirm a rigid formation and an adhesion to the substrate in the piers, a wall structure was also fabricated with only $3 \mathrm{MeV}$ proton beam. The pattern of the wall is similar to the lattice pattern of the pier. The wall was crossed the middle part of the girder between the piers.

The development was performed by immersing in diacetone alcohol at the room temperature without post-exposure baking. After the development, the sample was rinsed with isopropyl alcohol. Then, one of the drying processes was taken with the naturally drying in the air at room temperature. Another was taken with the supercritical drying. In the supercritical drying, the isopropyl alcohol was replaced by supercritical $\mathrm{CO}_{2}$ at $12 \mathrm{MPa}, 40{ }^{\circ} \mathrm{C}$, using a supercritical rinser $\&$ dryer, SCRD6, Rexxam Co., Ltd.

These structures were observed by SEM tilting the sample without any surface treatments.

\section{Results}

As a result of the naturally drying after the double exposure PBW, a flat bridge of epoxy resin was formed, as shown in Fig. 2(a). Figure 2(b) is magnified image of the center in Fig. 2(a). The height of the bridge is $20 \mu \mathrm{m}$, the same to the thickness of the coated SU-8 photoresist. The thickness of the girder is $8 \mu \mathrm{m}$, the almost same as the range of $0.5 \mathrm{MeV}$ proton. The bridge had the long span girder of $80 \mu \mathrm{m}$ making the space of 16 $\mu \mathrm{m}$ between the girder and the substrate.

The cavities, which were formed by the lattice pattern of the girder, have a concave shape. The width of the lattice beams was increased from 1.5 $\mu \mathrm{m}$ at the top to $3 \mu \mathrm{m}$ at the bottom.

The rigid formation of the long wall with the length of $500 \mu \mathrm{m}$ was confirmed. The height of the wall is $20 \mu \mathrm{m}$, the same to the pier height. From this result, the piers were also considered to be formed rigidly such as to be upstanding without the girder and to keep good adhesion to the substrate.

On the other hand, as a result of the supercritical drying, an arched bridge of epoxy resin was formed, as shown in Fig. 3(a). Figure 3(b) is magnified image of the center in Fig. 3(a). The girders between the piers were convex arched shape, while the girders at the piers were flat. The height of the top of convex girders is $6 \mu \mathrm{m}$ higher than flat girders, namely the height of arch bridge 

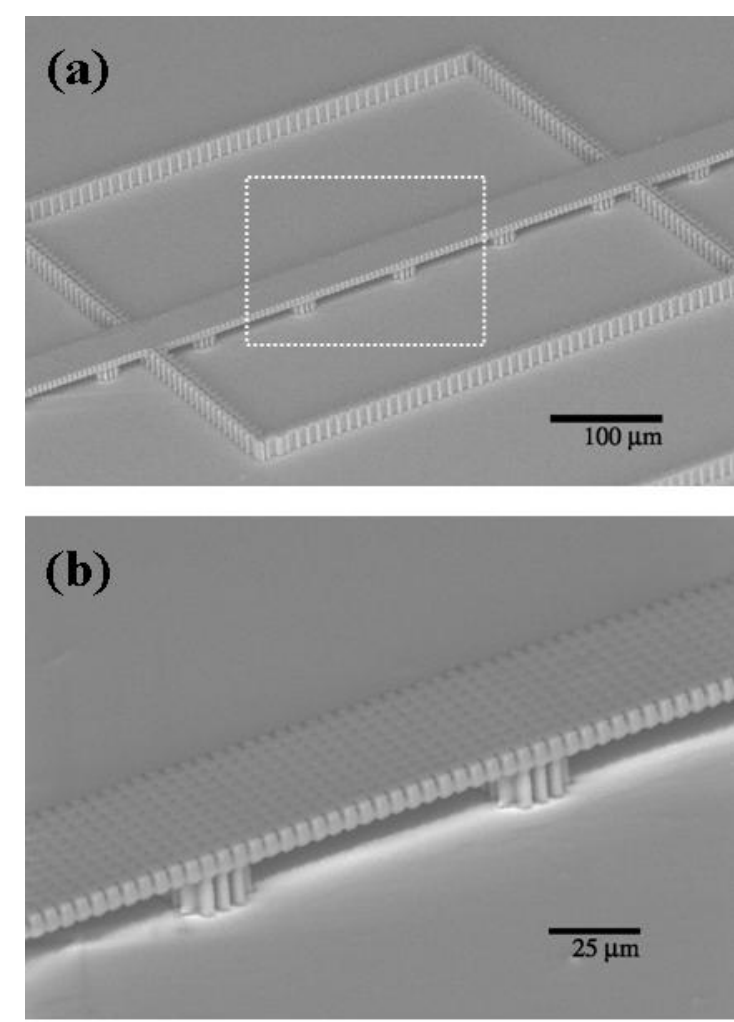

Fig. 2. SEM images of flat bridge and wall made from SU-8 photoresist. The flat epoxy bridge was fabricated by the combination of double exposure PBW with the incident energy of $0.5 \mathrm{MeV}$ and 3 $\mathrm{MeV}$ and natural drying in the air after the rinse with the isopropyl alcohol. The wall was fabricated with only $3 \mathrm{MeV}$ proton beam. (b) is the enlargement of the bridge, denoted by dashed rectangle in (a).

is $26 \mu \mathrm{m}$. Figure 4 shows side view of the arched bridge. A curve fitting to a side shape of the girder was executed by arcs, as shown in Fig. 4 . The inner and outer radius of the curvature at the top of arch is estimated to be approximately 79 and $72 \mu \mathrm{m}$. The length the outer arc is $9.7 \%$ longer than that of the inner arc. The curvature of the girder is $1.3 \times 10^{4}$ $1 / \mathrm{m}$. However, girders with the span of less than 35 $\mu \mathrm{m}$, denoted by solid lines in Fig. 4, kept flat.

The concave cavities at the middle parts of the girder filled such as the unevenness of the lattice pattern disappeared. On the writing with $0.5 \mathrm{MeV}$ proton beam, the deposition energy to the SU-8 photoresist increases from $47 \mathrm{keV} / \mu \mathrm{m}$ at the surface up to $101 \mathrm{keV} / \mu \mathrm{m}$ at the track end which is maximum of energy-deposition known as the
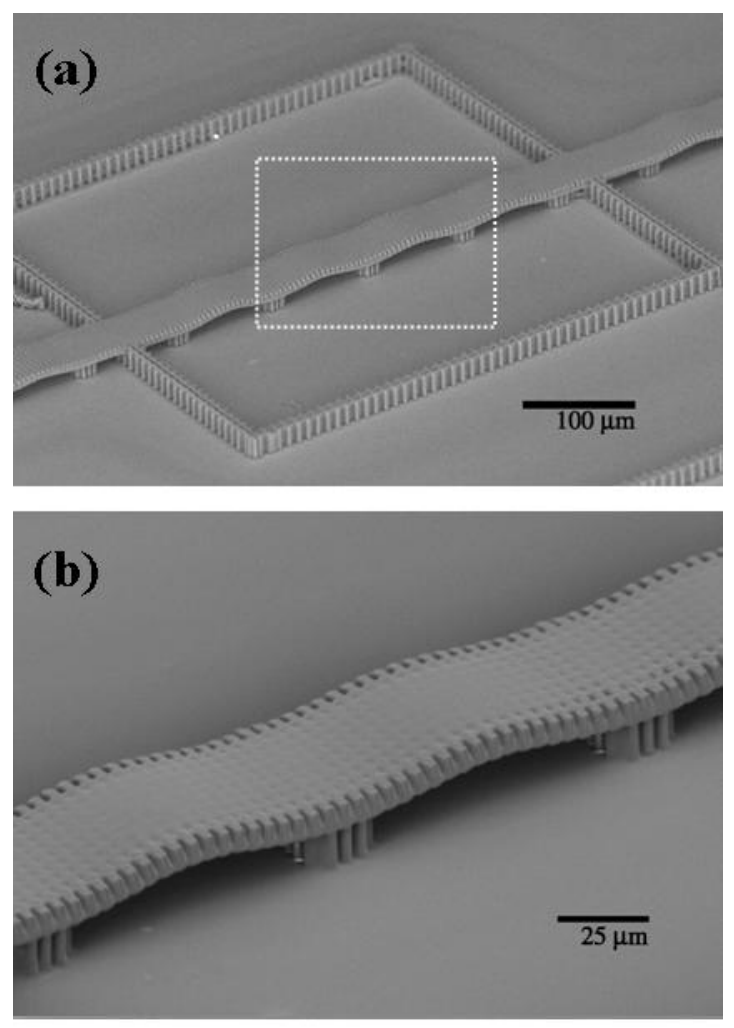

Fig. 3. SEM images of arched bridge and wall made from SU-8 photoresist. The arched bridge was fabricated by the combination of double exposure PBW with the incident energy of 0.5 $\mathrm{MeV}$ and $3 \mathrm{MeV}$ and supercritical drying method. The isopropyl alcohol as the rinse liquid was replaced by carbon dioxide drying at $12 \mathrm{MPa}$, $40{ }^{\circ} \mathrm{C}$. The wall was fabricated with only $3 \mathrm{MeV}$ proton beam. (b) is the enlargement of the bridge, denoted by dashed rectangle in (a).

Bragg peak. A lateral straggling on $0.5 \mathrm{MeV}$ proton passage is $0.3 \mu \mathrm{m}$. Therefore the beam-induced cross-linking reaction at the bottom of girder is promoted larger than that at the top of girder. The width increasing of the lattice beam is caused by spreading of the gelation area along the proton track. According to the spreading of the gelation region, the filling of cavities was also cased. On the supercritical drying method, a penetration of a solvent into the polymer matrix is considered to be induced. The penetration of the isopropyl alchohol to the low density region in the cross-linking is larger than that to the higher density. Therefore, the swelling at the upper of the girder is larger than that at the lower of the girder. Then, the curving of the girder was induced from the difference between 


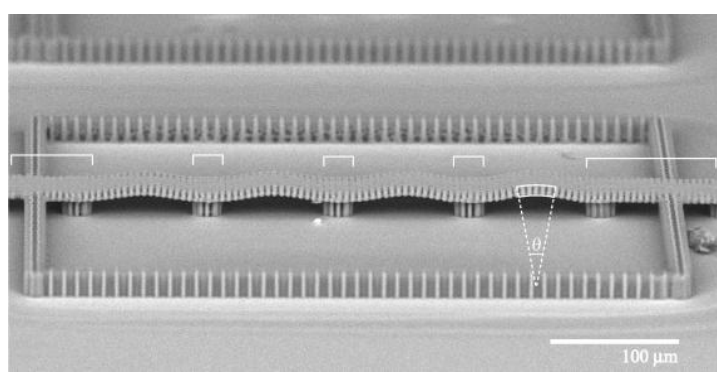

Fig. 4. Side view of arched bridge and wall made from SU-8 photoresist measured by SEM. Solid lines denote flat regions of the girder. Solid arcs are fitting curves to the top and bottom shapes of the girder. Dashed lines are inner and outer radius of the curvature. $\theta$ was taken to be 22 degree.

upper and lower girders in the swelling. However, in the case of shorter span of the less than $35 \mu \mathrm{m}$, the girder did not curved, because the difference between the top and the bottom of the girder in the swelled length is small.

In the case of supercritical drying, the wall was almost the same to that in the naturally drying, on the resolution of this measurement. It is concluded that the structures fabricated by the writing with $3 \mathrm{MeV}$ proton beam at the fluence of $4.5 \times 10^{3}$ ions $/ \mu \mathrm{m}^{2}$ did not almost swell, but kept to the shape, even then the supercritical drying. Therefore, the shape of the piers fabricated by the same condition in the writing with $3 \mathrm{MeV}$ proton beam should be almost the same to that in the naturally drying.

On the piers, the cross-linking density of the girder is higher than that between the piers, because the energy deposition was accumulated from girder writing with $0.5 \mathrm{MeV}$ proton beam and pier writing with $3 \mathrm{MeV}$ proton beam such as to form the rigid wall formation. Therefore, the girders on the pier and the wall written with by 3 $\mathrm{MeV}$ proton beam did not almost swell, but kept the flat shape.

Thus, utilized the curving of epoxy structure from the difference of swelling ratio between upper and lower of the girder at the supercritical drying, the fabrication of arched bridge of epoxy resin can be demonstrated by the combination of the double exposure PBW with different incident energy of the proton beam and the supercritical drying. The fabricated arched bridge has the span of $80 \mu \mathrm{m}$, the height of $26 \mu \mathrm{m}$, the curvature of $1.3 \times 10^{4} 1 / \mathrm{m}$, using SU-8 photoresist with the thickness of $20 \mu \mathrm{m}$. Unique curving technique in microprocessing was obtained by combination of the PBW and supercritical drying.

\section{Acknowledgments}

The authors would like to express their appreciation for the great cooperation of the staff in TIARA in carrying out the present experiments. They also acknowledge Koichi Miyake at Rexxam Co., Ltd. for the great cooperation at the execution of the supercritical drying. This work was supported by JSPS Funding Program for Next-Generation World-Leading Researches (NEXT Program) and KAKENHI from the MEXT Japan (No. 22226017, 19206105, and 17560625) from the Ministry of Education, Culture, Sports, Science and Technology, Japan.

\section{References}

1. S. V. Springham, T. Osipowicz, J. L. Sanchez, L. H. Gan, and F. Watt, Nucl. Instrum. Meth. B $\mathbf{1 3 0}$ (1997) 155.

2. J. A. van Kan, J. L. Sanchez, B. Xu, T. Osipowicz, and F. Watt,: Nucl. Instr. and Meth. B 148 (1999) 1085.

3. T. C. Sum, A. A. Bettiol, J. A. van Kan, F. Watt, E. Y. B. Pun, and K. K. Tung, Appl. Phys. Lett. 83 (2003) 1707.

4. F. Menzel, D. Spemann, S. Petriconi, J. Lenzner, and T. Butz, Nucl. Instr. and Meth. B 260 (2007) 419.

5. K. Takano, T. Satoh, Y. Ishii, M. Koka, T. Kamiya, T. Ohkubo, M. Sugimoto, H. Nishikawa, and S. Seki, Trans. Mater. Res. Soc. Jpn. 36 (2011) 305.

6. H. Namatsu, K. Yamazaki, and K. Kurihara, Microelectronic Engineering 46 (1999) 129.

7. H. Namatsu, K. Yamazaki, and K. Kurihara, $J$. Vac. Sci. Technol. B 18 (2000) 780.

8. M. Y. Lee, K. M. Do, H. S. Ganapathy, Y. S. Lo, J. J. Kim, S. J. Choi, and K. T. Lim, J. Supercritical Fluids 42 (2007) 150.

9. J. S. Chiou, J. W. Barlow, and D. R. Paul, J. Appl. Polym. Sci. 30 (1985)2633.

10. R. Mertsch and B. A. Wolf, Macromolecules 27 (1994) 3289.

11. T. Sakai, Y. Naitoh, T. Kamiya, and Y. Kobayashi, Nucl. Instr. and Meth. B 158 (1999) 250.

12. J. F. Ziegler, The Stopping and Ranges of Ions in Matter, Pergamon Press, Oxford, 197 
